Abstract PTU-078 Table 1 Plasma vitamin D response to differing doses of oral treatment in CD and UC

\begin{tabular}{|c|c|c|c|c|c|c|c|c|}
\hline & Subjects & $\begin{array}{l}\text { Vitamin D } \\
\text { deficient }(\%)\end{array}$ & $\begin{array}{l}\text { Treated orally }+ \text { follow up } \\
\text { Vitamin D available }\end{array}$ & $\begin{array}{l}\% \text { increase in } \\
\text { plasma vitamin D }\end{array}$ & High dose Rx & $\begin{array}{l}\% \text { increase in } \\
\text { plasma vitamin D }\end{array}$ & Low dose $R x$ & $\begin{array}{l}\% \text { increase in } \\
\text { plasma vitamin D }\end{array}$ \\
\hline All subjects & 205 & $95(46)$ & 32 & 115 & 24 & 150 & 8 & 34 \\
\hline UC & 70 & $35(50)$ & 11 & 100 & 8 & 167 & 3 & 47 \\
\hline All CD & 135 & $60(44)$ & 21 & 116 & 16 & 150 & 5 & 29 \\
\hline Ileocolonic CD & 45 & $20(44)$ & 9 & 132 & 7 & 156 & 2 & 13 \\
\hline Colonic CD & 36 & $15(42)$ & 5 & 64 & 3 & 114 & 2 & 46 \\
\hline Small bowel -CD & 54 & $25(46)$ & 7 & 145 & 6 & 173 & 1 & 14 \\
\hline
\end{tabular}

Conclusion There were no differences in plasma vitamin D concentrations between patients with CD and UC. Oral vitamin $\mathrm{D}$ replacement is an effective treatment for vitamin $\mathrm{D}$ deficiency in IBD patients and appears to be dose responsive; irrespective of whether patient have UC or CD (including small bowel disease). The optimal dose of oral vitamin $\mathrm{D}$ supplementation is yet to be determined, but higher doses are significantly more effective.

Disclosure of Interest None Declared

\section{PTU-079 THE INFLAMMATORY BOWEL DISEASE QUALITY IMPROVEMENT PROJECT - PROGRESS TWO YEARS ON}

doi:10.1136/gutjnl-2013-304907.171

1." I Shaw, ${ }^{2}$ E Fernandez on behalf of IBD OIP Steering Group. ${ }^{1}$ Department of Gastroenterology, Gloucestershire Royal Hospital, Gloucester; ${ }^{2}$ Clinical Standards Department, Royal College of Physicians, London, UK

Introduction The IBD Quality Improvement Project (IBDOIP) was established in 2010, as a project to help improve adult and paediatric IBD services across the UK. Here we report the first two years of progress.

Methods IBDOIP uses a web-based system (www.ibdqip.co.uk) to enable services to benchmark themselves against the national IBD standards and then action plan improvement using a combination of self-assessment, network support and a shared repository of best practise. Results are provided to sites graphically as a performance dashboard using a hierarchical grading system. Initial sites completed the first assessment in 2011, with a second round in 2012. Progress has been assessed by comparing scores between rounds and from site feedback.

Results Participation increased over time, with 69 sites (56 adult \& 13 paediatric) participating in round 1 (2011) and 84 sites (66 adult \& 18 paediatric) in round 2 (2012). $90 \%$ of sites completed their evaluation within 4 hours (median $2-3$ ). $60 \%$ of sites met as an MDT to complete their return, which was rated as the most powerful catalyst for change (figure 1). Significant improvement has been made across a range of measures:

\section{Abstract PTU-079 Table}

Sites with significant improvement in the year between 2011 and 2012 (for 44 adult sites completing both rounds)

Statements

$\mathrm{p}$ value

Written information about IBD and a range of treatments is made available $\quad 0.002$ to all patients

All IBD patients are provided with information about their local patient 0.039 support groups

$>50 \%$ IBD patients have regular stool chart documented during admission $\quad 0.008$ IBD patients can be referred by the IBD Service for psychological or 0.039 counselling input

Shared care agreement in place for management of stable patients 0.039 discharged back to primary care.

Patient surveys carried out annually 0.013

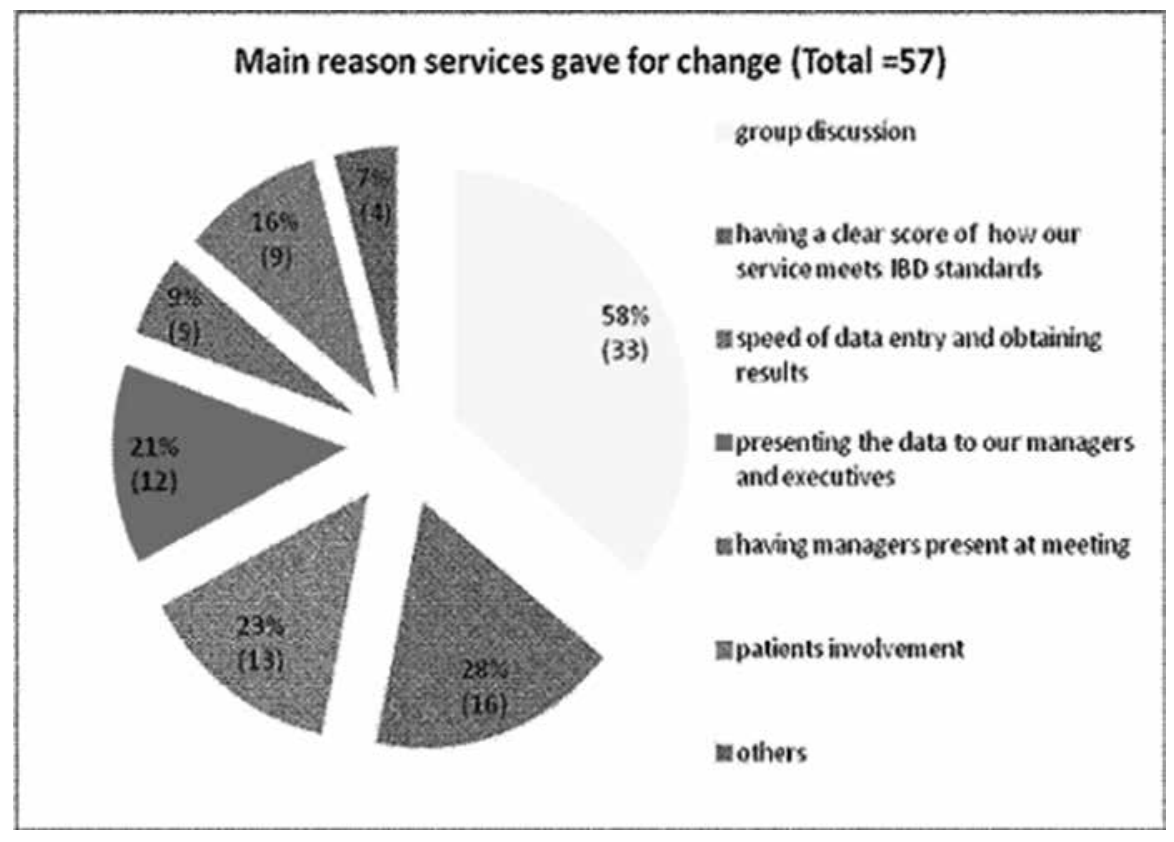


Conclusion In it's first two years, IBDOIP has shown increased participation and has proved to be a rapid and effective tool to improve IBD services. The project has now merged with the National IBD Audit and will be rolled out to all IBD services in 2014.

Disclosure of Interest None Declared

\section{PTU-080 DEVELOPMENT AND VALIDATION OF THE CROHN'S LIFE IMPACT QUESTIONNAIRE (CLIO)}

doi:10.1136/gutjnl-2013-304907.172

1."J Wilburn, 'S P McKenna, 'J Twiss, ${ }^{2} \mathrm{~K}$ Kemp, ${ }^{2} \mathrm{~S}$ Campbell. 'Galen Research Ltd; ${ }^{2}$ Department of Gastroenterology, Manchester Royal Infirmary, Manchester, UK

Introduction Crohn's Disease (CD) is a chronic, inflammatory, autoimmune disorder that substantially impairs patients' physical and emotional well-being. Despite this there is no CD-specific patient-reported outcome measure (PROM) available for determining the efficacy of alternative interventions for the condition. The objective of the study is to develop and validate the first such patient-reported outcome measure. Questionnaire content was derived from 30 qualitative interviews conducted with UK CD patients. Cognitive debriefing interviews conducted with a new sample of $15 \mathrm{CD}$ patients indicated that the draught scales were relevant, clear and easy to use.

Methods A test-retest postal survey was conducted to; identify the final scales, confirm their unidimensionality (by means of Rasch analysis) and to determine reproducibility and construct validity. A subset of the respondents was sent a second questionnaire package 2 weeks after completing the first. The package included the CLIO, the Nottingham Health Profile (NHP), the Unidimensional Fatigue Impact Scale (U-FIS) and a demographic questionnaire.

Results The questionnaire package was completed by $273 \mathrm{CD}$ patients (34.4\% male; aged 16-79 (mean: 43.9; SD 15.1) years). Of these, 107 also completed the second package. Items were removed from the scales that misfit the Rasch model $\left(\mathrm{Chi}^{2} \mathrm{p}>0.05\right)$, were redundant or displayed differential functioning by gender. Rasch analysis confirmed two unidimensional scales ( $p<0.05)$; activity limitations (11 items) and QoL (27 items). Internal consistency was good for both scales (0.93 and 0.91$)$ as was test-retest reliability (0.89 and 0.91 respectively). The CLIO scales were related (as expected) with the NHP section scores and the U-FIS. It was interesting to note that QoL scores were related to both physical and emotional impairments.

Conclusion The CLIO is the first scientifically rigorous PROM designed specifically for CD patients. It consists of two unidimensional scales with excellent psychometric properties. It should prove to be a valuable tool for evaluating the impact of $\mathrm{CD}$ and its treatment from the patients' perspective.

Disclosure of Interest None Declared

\section{PTU-081 ARE WE EXPOSING PATIENTS WITH A MILDLY ELEVATED FAECAL CALPROTECTIN TO UNNECESSARY INVESTIGATIONS?}

doi:10.1136/gutjnl-2013-304907.173

1."J P Seenan, '1F Thomson, ${ }^{2 K}$ Rankin, ${ }^{2 K}$ Smith, 'D R Gaya. 'Gastroenterology; ${ }^{2}$ Biochemistry, Glasgow Royal Infirmary, Glasgow, UK

Introduction Faecal calprotectin (FC) is increasingly used as a non-invasive marker to differentiate irritable bowel syndrome (IBS) from inflammatory bowel disease (IBD). However, it is a non-specific marker of luminal inflammation and false positives are common. We have previously demonstrated a low yield of diagnostic colonoscopy in patients with borderline elevations of FC $(50-100 \mu \mathrm{g} / \mathrm{g})$. Higher FC levels $(100-200 \mu \mathrm{g} / \mathrm{g})$ often prompt more extensive investigation. We sought to determine the diagnostic yield of endoscopic/radiological investigation in patients presenting with new lower GI symptoms and a mildly elevated FC (100-200 $\mu \mathrm{g} / \mathrm{g})$.

Methods All patients with a faecal calprotectin 100-200 $\mu \mathrm{g} / \mathrm{g}$ were identified from our biochemistry laboratory database between September 2009 and September 2011. Patients aged 16 to 50 years attending gastroenterology outpatient clinics with new lower GI symptoms were identified. Patients were excluded if they had a previous $\mathrm{FC}>200 \mu \mathrm{g} / \mathrm{g}$, were taking NSAIDs, had known IBD, positive stool cultures or any 'alarm' symptoms. Details of investigations, diagnosis and clinical outcomes were determined electronically from the NHS Greater Glasgow and Clyde Clinical Portal.

Results 163 patients (104 female) were identified who met the inclusion criteria. The mean age was 37.5 years with a mean FC of $146.6 \mu \mathrm{g} / \mathrm{g}$. The primary presenting complaint was diarrhoea in 100 $(61.3 \%)$ and abdominal pain in $63(38.7 \%)$. Secondary symptoms were abdominal pain $(28.2 \%)$, diarrhoea $(18.4 \%)$ and constipation (1.8\%). A total of 390 endoscopic, radiological and histological investigations were undertaken in 152 patients with an average of 2.6 investigations per patient. 131 colonoscopies were performed with abnormalities detected in only 23 (17.6\%). In patients with a macroscopically normal upper GI endoscopy and colonoscopy, the diagnostic yield of any further investigation was only $7 \%$. The negative predictive value (NPV) of a FC $100-200 \mu \mathrm{g} / \mathrm{g}$ was $86.9 \%$ for any pathology and $98.1 \%$ for significant luminal pathology (IBD, advanced adenoma or colorectal carcinoma). IBD was the final diagnosis in only $3(1.8 \%)$ of patients while $48.5 \%$ were diagnosed as having IBS.

Conclusion In adult patients under 50 years old presenting with new lower GI symptoms, the NPV of a FC between 100 and $200 \mu \mathrm{g} / \mathrm{g}$ in excluding significant organic GI disease is high. Patients are often extensively investigated yet the overall diagnostic yield is very low and the majority of these patients have functional disease. We suggest that the manufacturer's FC cut off of $50 \mu \mathrm{g} / \mathrm{g}$ of stool is too low for utilisation in clinical practise and often results in unnecessary, invasive investigations.

Disclosure of Interest None Declared

\section{PTU-082 FAECAL INCONTINENCE IN INFLAMMATORY BOWEL DISEASE: WE DON'T ASK AND THEY DON'T TELL}

doi:10.1136/gutjnl-2013-304907.174

1,"J Duncan, ${ }^{1} \mathrm{G}$ Sebepos-Rogers, ${ }^{2} \mathrm{O}$ Poole-Wilson, ${ }^{3} \mathrm{C}$ To, ${ }^{4} \mathrm{~J}$ B Canavan, ${ }^{1} \mathrm{~V}$ Kariyawasam, 'M Ward, 'R Goel, ' $\mathrm{K}$ Patel, 'A Stanton, 'M Sastrillo, 'S Anderson, ' $\mathrm{K}$ Taylor, 1J Sanderson, 'P Irving. 'Gastroenterology, Guy's \& St Thomas' NHS Foundation Trust; ${ }^{2}$ South London Healthcare Trust, London, UK; ${ }^{3}$ Australian National University, Canberra, Australia; ${ }^{4}$ Kings College London, London, UK

Introduction The deleterious effect of faecal incontinence (FI) on quality of life (QOL) is well documented. People with FI experience stigma, embarrassment and social exclusion, and report adverse effects on activities and relationships. Restoration of continence is associated with improvement in QOL. Diarrhoea is associated with increased prevalence of FI and, therefore, people with inflammatory bowel disease (IBD) are at risk.

Methods To investigate how frequently health care professionals (HCPs) assess FI in a cohort of patients with IBD we performed a cross sectional survey of 380 adults attending a tertiary referral IBD clinic. Patient surveys were: the validated ICIO-B questionnaire, detailing frequency and severity of bowel pattern, control and quality of life; and the non-validated Bowel Leakage Questionnaire, detailing any prior interventions by health care professionals. Demographics of age, gender, diagnosis, Montreal classification, St Mark's Continence Score and disease activity were also recorded. Data was entered into a database and analysed using SPSS statistical package. Results 229/380 (60\%) had Crohn's Disease (CD) and 180/380 (47\%) were female. Median age was 38 years (IOR:31-50) with a median disease duration of 8.7 years (3.4-15.1). $343 / 380$ (90\%) had 